\title{
Erratum to: Welfare States and Social Support: An International Comparison
}

\author{
Livia García-Faroldi
}

Published online: 23 July 2014

(C) Springer Science+Business Media Dordrecht 2014

\section{Erratum to: Soc Indic Res \\ DOI 10.1007/s11205-014-0671-1}

Two small corrections about the tables the author had suggested in the proofs have not been included.

First, in Table 5, the Word COUNTRIES is in capital letter, instead of in lower case letter, as it appears in Tables 3 and 4. Second, in Tables 3, 4 and 5 the words Individual variables are in the upper part of the table with the categories of the dependent variables, but they should be included below, in the first row with the independent variables.

The tables should read:

The online version of the original article can be found under doi:10.1007/s11205-014-0671-1.

L. García-Faroldi $(\bowtie)$

Department of Sociology, Faculty of Communication, University of Málaga, Campus de Teatinos, 29071 Málaga, Spain

e-mail: lgarcia@uma.es 
Table 3 Marginal effects of the probability of asking for domestic help among interviewees with a partner, both parents, or the mother living, and with close friends: multinomial logit model

\begin{tabular}{|c|c|c|c|}
\hline & Partner & Mother & Offspring \\
\hline \multicolumn{4}{|l|}{ Individual variables } \\
\hline Sex (ref. woman) & $0.088 * * *$ & $-.054 * * *$ & $-.034 * * *$ \\
\hline \multicolumn{4}{|l|}{ Age (ref. 35-44) } \\
\hline \multicolumn{4}{|l|}{$18-24$} \\
\hline $25-34$ & & & $-.029 * * *$ \\
\hline $45-54$ & $0.033 * * *$ & $-.054 * * *$ & $0.021 * * *$ \\
\hline $55-64$ & $0.095^{* * *}$ & $-.112 * * *$ & $0.018 * *$ \\
\hline \multicolumn{4}{|l|}{65 and +} \\
\hline \multicolumn{4}{|c|}{ Education (ref. secondary) } \\
\hline Primary & & & $0.014 * *$ \\
\hline University & & $0.169 * *$ & $-0.18 * * *$ \\
\hline \multicolumn{4}{|c|}{ Residence (ref. place of birth) } \\
\hline \multicolumn{4}{|l|}{ Less than 5 years } \\
\hline More than 5 years & & & $0.011 * *$ \\
\hline \multicolumn{4}{|l|}{ Habitat (ref. urban) } \\
\hline \multicolumn{4}{|l|}{ Rural } \\
\hline \multicolumn{4}{|c|}{ Religious practice (ref. infrequent observance) } \\
\hline Nonobservant & $-.017^{*}$ & $0.024 * *$ & \\
\hline \multicolumn{4}{|l|}{ Medium observance } \\
\hline \multicolumn{4}{|l|}{ High observance } \\
\hline \multicolumn{4}{|l|}{ Network } \\
\hline \multicolumn{4}{|l|}{ Visit/see sibling } \\
\hline Visit/see father & & $0.002 * *$ & \\
\hline Visit/see mother & $-.034 * * *$ & $0.034 * * *$ & \\
\hline \multicolumn{4}{|c|}{ Size network of friends } \\
\hline \multicolumn{4}{|l|}{ Countries (ref. Spain) } \\
\hline Great Britain & $0.141^{* * *}$ & $-.087 * * *$ & $-.054 * *$ \\
\hline USA & $0.069^{* * *}$ & $-.038 * * *$ & $-.031 * *$ \\
\hline Canada & $0.109^{* * *}$ & $-.087 * * *$ & $-.021 * *$ \\
\hline New Zealand & $0.170^{* * *} *$ & $-.138 * * *$ & $-.032 * * *$ \\
\hline Austria & $0.043^{* * *}$ & $-.029 * *$ & $-.014 *$ \\
\hline West-Germany & $0.044 * * *$ & & $-.030 * * *$ \\
\hline East-Germany & $0.090 * * *$ & $-.078 * * *$ & \\
\hline France & $0.075^{* * *}$ & $-.049^{* * *}$ & $-.025 * * *$ \\
\hline Norway & $0.165^{* * * *}$ & $-.131 * * *$ & $-.034 * * *$ \\
\hline Denmark & $0.101 * * *$ & $-.061 * * *$ & $-.039 * * *$ \\
\hline Finland & $0.163^{* * *} *$ & $-.132 * * *$ & $-.030 * * *$ \\
\hline Italy & & $0.029 * * *$ & \\
\hline Log likelihood & $-1,215.0339$ & & \\
\hline Likelihood ratio test & $1,581.58 * * *$ & & \\
\hline Pseudo- $R^{2}$ & 0.394 & & \\
\hline No. observations & 4,761 & & \\
\hline
\end{tabular}

Sig. $* p \leq 0.1 ; * * p \leq 0.05 ; * * * p \leq 0.01$. Source: ISSP (2001) 
Table 4 Marginal effects of the probability of asking for economic help among interviewees with a partner, both parents, or the mother living, and with close friends: multinomial logit model

\begin{tabular}{|c|c|c|c|}
\hline & Partner & Parents & Siblings \\
\hline \multicolumn{4}{|l|}{ Individual variables } \\
\hline Sex (ref. woman) & $-.171 * * *$ & $0.135 * * *$ & $0.037 * * *$ \\
\hline \multicolumn{4}{|l|}{ Age (ref. 35-44) } \\
\hline $18-24$ & $-.170 * * *$ & $0.207 * * *$ & $-.038 *$ \\
\hline $25-34$ & $-.064 * * *$ & $0.082 * * *$ & \\
\hline $45-54$ & $0.050 * *$ & $-.065^{* * *}$ & \\
\hline $55-64$ & $0.205 * * *$ & $-.218 * * *$ & \\
\hline 65 and + & $0.524 * * *$ & $-.563 * * *$ & \\
\hline \multicolumn{4}{|l|}{ Education (ref. secondary) } \\
\hline \multicolumn{4}{|l|}{ Primary } \\
\hline University & & & $-.026^{* *}$ \\
\hline \multicolumn{4}{|c|}{ Residence (ref. place of birth) } \\
\hline Less than 5 years & & & $0.027 *$ \\
\hline \multicolumn{4}{|l|}{ More than 5 years } \\
\hline \multicolumn{4}{|l|}{ Habitat (ref. urban) } \\
\hline \multicolumn{4}{|l|}{ Rural } \\
\hline \multicolumn{4}{|c|}{ Religious practice (ref. Infrequent observance) } \\
\hline \multicolumn{4}{|l|}{ Nonobservant } \\
\hline \multicolumn{4}{|l|}{ Medium observance } \\
\hline \multicolumn{4}{|l|}{ High observance } \\
\hline \multicolumn{4}{|l|}{ Network } \\
\hline Visit/see sibling & $0.010 *$ & $-.028 * * *$ & $0.018 * * *$ \\
\hline Visit/see father & $-.013 * * *$ & $0.024 * * *$ & $-.011 * * *$ \\
\hline Visit/see mother & $-.035^{* * *}$ & $0.040 * * *$ & \\
\hline Size network of friends & $0.001 *$ & & $-.001 * *$ \\
\hline \multicolumn{4}{|l|}{ Countries (ref. Spain) } \\
\hline Great Britain & $0.089 *$ & & \\
\hline USA & $-.114 * *$ & $0.098 * *$ & \\
\hline \multicolumn{4}{|l|}{ Canada } \\
\hline \multicolumn{4}{|l|}{ New Zealand } \\
\hline Austria & $0.151 * * *$ & $-.097 * *$ & $-.053 *$ \\
\hline \multicolumn{4}{|l|}{ West-Germany } \\
\hline East-Germany & $-.121 * *$ & $0.164 * * *$ & \\
\hline France & & $0.090 * *$ & $-.041^{*}$ \\
\hline Norway & $0.122 * * *$ & & $-.066 * * *$ \\
\hline Denmark & & & $-.069 * *$ \\
\hline Finland & $0.184 * * *$ & $-.158 * * *$ & \\
\hline Italy & $-.152 * * *$ & $0.117 * * *$ & $0.034 *$ \\
\hline Log likelihood & $-2,155.596$ & & \\
\hline Likelihood ratio test & $730.74 * * *$ & & \\
\hline Pseudo- $\mathrm{R}^{2}$ & 0.145 & & \\
\hline No. observations & 2,847 & & \\
\hline
\end{tabular}

Sig. $* p \leq 0.1 ; * * p \leq 0.05 ; * * * p \leq 0.01$. Source: ISSP (2001) 
Table 5 Marginal effects of the probability of asking for emotional help among interviewees with a partner, both parents, or the mother living, and with close friends: multinomial logit model

\begin{tabular}{|c|c|c|c|}
\hline & Partner & Mother & Close friend \\
\hline \multicolumn{4}{|l|}{ Individual variables } \\
\hline Sex (ref. woman) & $0.177 * * *$ & $-.060 * * *$ & $-.117 * * *$ \\
\hline \multicolumn{4}{|l|}{ Age (ref. 35-44) } \\
\hline $18-24$ & $-.065 * * *$ & $0.038 * * *$ & \\
\hline $25-34$ & & $0.016^{*}$ & $-.027 *$ \\
\hline $45-54$ & $0.029 *$ & & \\
\hline $55-64$ & $0.115^{* * *}$ & $-.055^{* *}$ & $-.060 * *$ \\
\hline 65 and + & $0.352 * *$ & & $-.345^{* *}$ \\
\hline \multicolumn{4}{|c|}{ Education (ref. secondary) } \\
\hline \multicolumn{4}{|l|}{ Primary } \\
\hline University & & $-.021 * * *$ & \\
\hline \multicolumn{4}{|c|}{ Residence (ref. place of birth) } \\
\hline Less than 5 years & $0.061 * * *$ & & $-.057 * * *$ \\
\hline \multicolumn{4}{|l|}{ More than 5 years } \\
\hline \multicolumn{4}{|l|}{ Habitat (ref. urban) } \\
\hline \multicolumn{4}{|l|}{ Rural } \\
\hline \multicolumn{4}{|c|}{ Religious practice (ref. infrequent observance) } \\
\hline Nonobservant & $-.073 * * *$ & & $0.071 * * *$ \\
\hline \multicolumn{4}{|l|}{ Medium observance } \\
\hline High observance & $0.049 * *$ & & \\
\hline \multicolumn{4}{|l|}{ Network } \\
\hline \multicolumn{4}{|l|}{ Visit/see sibling } \\
\hline Visit/see father & & $0.005^{* *}$ & \\
\hline Visit/see mother & $-.024 * * *$ & $0.012 * * *$ & $0.012 * *$ \\
\hline Size network of friends & $0.002 * *$ & $-.002 * * *$ & \\
\hline \multicolumn{4}{|l|}{ Countries (ref. Spain) } \\
\hline \multicolumn{4}{|l|}{ Great Britain } \\
\hline USA & $-.212 * * *$ & $-.044 * * *$ & $0.168 * * *$ \\
\hline Canada & $-.061 *$ & $-.039 *$ & $0.100 * * *$ \\
\hline \multicolumn{4}{|l|}{ New Zealand } \\
\hline \multicolumn{4}{|l|}{ Austria } \\
\hline West-Germany & & & $0.078 * *$ \\
\hline \multicolumn{4}{|l|}{ East-Germany } \\
\hline France & $-.141 * * *$ & & $0.125 * * *$ \\
\hline Norway & & $-.045^{* *}$ & \\
\hline Denmark & & $-.043 * *$ & \\
\hline Finland & & $-.047 * *$ & \\
\hline Italy & $-.264 * * *$ & & $0.265^{* * *}$ \\
\hline Log likelihood & $-2,762.6373$ & & \\
\hline Likelihood ratio test & $768.88 * * *$ & & \\
\hline Pseudo-R ${ }^{2}$ & 0.122 & & \\
\hline No. observations & 4,344 & & \\
\hline
\end{tabular}

Sig. * $p \leq 0.1 ; * * p \leq 0.05 ; * * * p \leq 0.01$. Source: ISSP (2001) 